\section{AIDS and tuberculosis in medical inpatients in Malawi}

MM Weismuller, S Meijnen, NJM Claessens, FM Salaniponi, AD Harries

\section{Introduction}

Malawi is gripped by a twin epidemic of AIDS and tuberculosis (TB). In 1999, the country had an estimated 800,000 people living with HIV/AIDS1, and 24,396 registered cases of TB (source = National Tuberculosis Programme). Health workers have little hesitation in considering TB as a possible diagnosis, but the same cannot be said of AIDS. AIDS is still a highly stigmatised disease, and there seems to be reluctance by health workers to label patients with this diagnosis or refer patients for voluntary counselling and HIV testing (VCT). We carried out a countrywide cross-sectional survey to document i) the principal working diagnoses in medical in-patients, ii) the frequency with which TB was considered in the differential diagnosis and investigated by sputum smear examination and iii) how often AIDS or a synonym of AIDS was written in the case file or a referral made for VCT.

\section{Methods}

All 44 district, mission and central hospitals in Malawi where TB registration and treatment takes place were visited between April and June 2001. In each hospital, all adult non-fee paying medical in-patients aged 15 years and above who were in general or medical wards were seen. Case notes and treatment cards were inspected, and data related to the study objectives were collected into structured proformas. Patients who were already registered and receiving treatment for TB were not included. Data were analyzed using EPI-INFO 6.04 software. Proportions were compared using $\mathrm{X} 2$ test, differences at the $5 \%$ level being regarded as significant.

\section{Results}

There were 1536 patients, 695 men and 841 women, whose mean age (SD) was 37 (14) years. The top 10 working diagnoses in men, women, and all patients, are shown in the Table.
TB was the most common principal working diagnosis, while AIDS was fourth. In $614(40 \%)$ patients, TB was considered either as the principal diagnosis or in the differential diagnosis, and in $572(37 \%)$ patients sputum specimens had been requested for AFB examination: there were no differences between men and women.

In $223(15 \%)$ patients, AIDS or one of its synonyms was mentioned in the case files (this included patients with a principal diagnosis of AIDS), and in $138(9 \%)$ VCT had been requested: there were no differences between men and women. Fifty six patients had a principal working diagnosis of Kaposi's Sarcoma, chronic enteropathy, cryptococcal meningitis, Pneumocystis carinii pneumonia, or oesophageal candidiasis: AIDS was mentioned in $24(43 \%)$ and VCT requested in $20(36 \%)$ of these patients. Three hundred and seven patients had a principal working diagnosis of TB: AIDS was mentioned in 43 (14\%) and VCT requested in $26(9 \%)$. AIDS was mentioned in the case files more frequently in mission (21\%) and central $(17 \%)$ hospitals compared with district $(11 \%)$ hospitals $[\mathrm{p}<0.05]$. Referrals for VCT were low in each type of hospital (mission - 11\%; central $11 \%$; and district $-8 \%$ ).

\section{Discussion}

This cross-sectional study highlights the enormous burden imposed on hospitals by the TB epidemic. Many patients are considered for possible TB, and in over one-third sputum specimens are being requested for laboratory AFB examination. Seventy seven percent of TB patients in Malawi are HIVseropositive2. In Queen Elizabeth Central Hospital, over $70 \%$ of all medical in-patients are HIV-seropositive (Zijlstra, personal communication), in keeping with findings from other African countries3. Despite this, AIDS is infrequently written in case files, even when diseases which are almost always associated with HIV, such as Kaposi's Sarcoma or cryptococcal meningitis, are diagnosed. There are few referrals for VCT, even in patients with suspected TB or a classical HIV-related disease.

Stigma and the absence of anything to offer a patient labeled with a diagnosis of AIDS are the likely explanations for not mentioning the diagnosis of AIDS in case notes or referring patients for VCT. However, changes have to take place if Malawi is start winning the battle against HIV / AIDS. Modeling studies in East Africa have demonstrated the cost-effectiveness

\begin{tabular}{|c|c|c|c|c|c|c|c|c|}
\hline Male & & & Female & & & Total & & \\
\hline Diagnosis & No. & $(\%)$ & Diagnosis & No. & $(\%)$ & Diagnosis & No. & $(\%)_{-}$ \\
\hline Tuberculosis* & 127 & $(18.3)$ & Tuberculosis** & 180 & $(21.4)$ & Tuberculosis*** & 307 & $(20.0)$ \\
\hline Pneumonia & 116 & $(16.7)$ & Pneumonia & 168 & $(20.0)$ & Pneumonia & 284 & $(18.5)$ \\
\hline Malaria & 72 & $(10.4)$ & Malaria & 109 & $(13.0)$ & Malaria & 181 & (11.8) \\
\hline AIDS & 39 & $(5.6)$ & AIDS & 38 & $(4.5)$ & AIDS & 77 & $(5.0)$ \\
\hline Anaemia & 25 & $(3.6)$ & Anaemia & 38 & $(4.5)$ & Anaemia & 63 & $(4.1)$ \\
\hline Heart failure & 25 & $(3.6)$ & Gastro-enteritis & 32 & $(3.8)$ & Heart failure & 50 & (3.3) \\
\hline Meningitis & 24 & (3.5) & PID & 25 & $(3.0)$ & Gastro-enteritis & 49 & $(3.2)$ \\
\hline Stroke & 19 & $(2.7)$ & Heart failure & 25 & (3.0) & Meningitis & 45 & (2.9) \\
\hline Ascites & 19 & $(2.7)$ & Meningitis & 25 & $(2.5)$ & Ascites & 40 & (2.6) \\
\hline Bacteraemia & 17 & $(2.4)$ & Ascites & 21 & $(2.5)$ & Bacteraemia & 37 & (2.4) \\
\hline Total & 483 & $(69.5)$ & & 657 & $(78.2)$ & & 1133 & $(73.8)$ \\
\hline * No. PTB 113 & & ! & ** No. PTB 164 & & & **** No. PTB 277 & & \\
\hline No. EPTB 14 & & : & No. EPTB 16 & & & No. EPTB 30 & & \\
\hline
\end{tabular}

Legend: PID = Pelvic Inflammatory Disease PTB $=$ Pulmonary TB EPTB $=$ Extrapulmonary TB 
of VCT in averting further HIV infections4. Cotrimoxazole prophylaxis, recommended by UNAIDS as part of a minimum package of care for people living with AIDS in Africa5, should be considered for HIV-seropositive patients, and this may provide individual benefit. Antiretroviral therapy may become accessible to the population in the future. Health workers must take a lead in this difficult area, and can begin by "breaking the silence".

Acknowledgements

We thank the Department for International Development (DFID), UK, the Norwegian Agency for Development Cooperation (NORAD) and the Royal Dutch Tuberculosis Association (KNCV) for financial support as part of their aid contribution to Operational Research of the Malawi National Tuberculosis Control Programme. The study received the approval of the National Health Science Research Committee.

This article has previously been published in Tropical Doctor in 2002

Authors:

Menco M Weismuller1, Sander Meijnen1, Niels JM Claessens1, Felix M

Salaniponi MSc PhD2, Anthony D Harries MD FRCP2

1 Academic Medical Centre, University of Amsterdam, Amsterdam, The Netherlands

2 National Tuberculosis Control Programme, Ministry of Health, PO Box 30377, Capital City, Lilongwe 3, Malawi

Address for Correspondence:

Professor AD Harries, c/o British High Commission, PO Box 30042 ,

Lilongwe 3, Malawi

Fax: (265) 772 657; Email: adharries@malawi.net

References

1. UNAIDS. Malawi - epidemiological fact sheet on HIV/AIDS and sexually transmitted infections. 2000 Update. UNAIDS and World Health Organization.

2. Kwanjana JH Harries AD Gausi F Nyangulu DS Salaniponi FM. TB-HIV seroprevalence in patients with tuberculosis in Malawi. Malawi Medical Journal 2001; 13: $7-10$.

2. Grant AD Sidibe K Domoua K et al. Spectrum of disease among HIV-infected adults hospitalised in a respiratory medicine unit in Abidjan, Cote d'Ivoire. Int J Tuberc Lung Dis $1998 ; 2: 926-934$.

4. Sweat M Greogorich S Sangiwa G et al. Cost-effectiveness of voluntary HIV-1 counselling and testing in reducing sexual transmission of HIV-1 in Kenya and Tanzania Lancet 2000; 356: $113-121$.

3. UNAIDS. Provisional WHO/UNAIDS Secretariat recomnendations on the use of cotrimoxazole prophylaxis in adults and children living with HIV/AIDS in Africa. UNAIDS / WHO 2000.

\section{The impact of HIV infection on childhood pneumonia: compari- son between developed and devel- oping regions}

\author{
Stephen M Graham
}

\section{Summary}

Respiratory disease is the commonest cause of morbidity and mortality in HIV-infected children. While the pattern of HIV-related pneumonia in African adults is well documented and is recognised as quite different from that which occurs among HIV-infected adults in high-income regions, less is known of the situation in children. Most children are infected by mother-to-child transmission and presentation of HIV-related pneumonia is often in infancy or early childhood, an age group in which confirmation of the cause of pneumonia is difficult. However, aetiological data are important. Poor response of the infant with severe pneumonia to standard antibiotic (such as chloramphenicol) or of the older child with chronic pneumonia to anti-tuberculosis treatment are two very common clinical dilemmas that many Malawian health workers would recognise. This review aims to present the available data relevant to Malawi, contrast with experience from the developed world and to describe common HIV-related pneumonias such as PCP and LIP. Unlike for adults, the pattern of HIV-related pneumonia in Malawian children may not be so different in cause from that described for children in developed countries prior to the use of PCP prophylaxis and anti-retroviral therapies. The most important contrast is the higher prevalence and poorer outcome.

\section{Introduction}

Respiratory disease is a major cause of morbidity and mortality in HIV-infected children in developed and developing countries. ${ }^{1-3}$ Prior to the HIV epidemic, there were already important differences between the regions that still exist. In developing countries, acute childhood pneumonia is more often due to bacteria, most commonly Streptococcus pneumoniae and Haemophilus influenzae, and more likely to be fatal. ${ }^{4}$ The prevalence is much higher of important risk factors for pneumonia morbidity and mortality such as fetal and early childhood malnutrition. In addition, the community prevalence of smear-positive pulmonary tuberculosis (PTB) has increased dramatically in HIV endemic regions and maternal illness and death is common. It is therefore a difficult environment for an HIV-infected child to negotiate and perhaps not surprising that the majority of HIVinfected infants from a resource-poor region such as tropical Africa have died by 3 years of age. ${ }^{5,6}$ Only about $25 \%$ survive up to 5 years compared to over $80 \%$ in USA or western Europe. 1 Thus the majority of cases of HIV-related respiratory disease present in infancy and early childhood. However, because childhood HIV infection is so common in some countries, the presentation of HIV-infected school-aged children often with chronic respiratory disease is not unusual.

Causes of pneumonia in HIV-infected children living in highincome countries such as USA or UK are well documented. ${ }^{3,8}$ It may not be correct to assume that the pattern of disease is similar in HIV-infected children in resource-poor Africa where it is estimated that over $90 \%$ of childhood HIV infection now 